\title{
Improved microscopy diagnosis of pulmonary tuberculosis using sodium hypochlorite concentration technique in Tanga, Tanzania
}

\author{
W.H. MAKUNDE ${ }^{1 *}$, R.A. MAKUNDE ${ }^{2}$, L.M. KAMUGISHA ${ }^{1}$, S.G. MGEMA ${ }^{3}$ and A. LIWA ${ }^{4}$ \\ ${ }^{1}$ National Institute for Medical Research, Tanga Centre, P.O. Box 5004, Tanga, Tanzania \\ ${ }^{2}$ Tanzania Public Health Association, Dar es Salaam, Tanzania \\ ${ }^{3}$ Lushoto District Hospital, Lushoto, Tanzania \\ ${ }^{4}$ Mikocheni Mission Hospital, Dar es Salaam, Tanzania
}

\begin{abstract}
Pulmonary tuberculosis diagnosis commonly relies on the bacteriological examination of sputum. A cross-sectional hospital-based study was carried out to compare on "on the spot" sputum staining using sodium hypochlorite (bleach method) and routine Ziel-Neelsen (ZN) staining technique. Study candidates included individual patients presenting with cough $<3$ weeks (Group I) and $\geq 3$ weeks (Group II). Sensitivity and specificity of the bleach method was calculated and compared at $100 \%$ using the ZN staining technique as the standard. A total of 171 patients (94 males, 77 females) with mean age 34.9 years $(S D \pm 12.9)$ were recruited. Fifty-eight patients had coughed for $<3$ weeks while 113 had coughed for $\geq 3$ weeks. Smear-positive TB in Group I was $13.8 \%$ (95\% CI = 5-23) while in Group II was 25.7\% (95\% CI = 21-29). Using the bleach method, the prevalence of smear-positive TB in Group II was $28.3 \%$ (CI 95\% = 20-36). This was an increase in smear-positivity rate of $15.6 \%$ as compared to the $\mathrm{ZN}$ technique. These results suggest that the use of bleach technique "on the spot" improve the sensitivity of tuberculosis diagnosis among patients with a history of coughing of over three weeks. However, further studies in different settings are recommended to validate the technique.
\end{abstract}

Key words: pulmonary tuberculosis, diagnosis, bleach, hypochlorite, Ziel-Neelsen, Tanzania

\section{Introduction}

Tuberculosis (TB) is a growing threat and a global health burden especially in developing world. The disease was declared a global emergency by World Health Organization since 1993. In 2003, 8.8 million new cases of TB were reported worldwide with 1.7 million deaths. Eight per cent of the new cases and $13.5 \%$ of the deaths were among individuals infected with HIV (WHO, 2006). In majority of developing countries, TB case-finding depends on self-referred of symptomatic individuals who attend health facilities and are then subsequently diagnosed as suffering from tuberculosis (L'Herminez, 1993). Sputum microscopy is the mainstay technique for the diagnosis of pulmonary tuberculosis as it is the most reliable, specific and objective method available especially in developing world. The current strategy for diagnosing pulmonary TB is to screen sputum for Mycobacterium tuberculosis of patients presenting with history of a cough for more than two weeks. However, many settings including Tanzania have not been able to put this new strategy fully function in their routine services. The use of the old strategy of screening sputum of patients presenting with history of cough for more than 3 weeks in still common among a number of practitioners in Tanzania.
The cut off point of more than three weeks is too long and likely to contribute to patients to seek treatment outside the conventional healthcare facility. Moreover, this cut-off point leaves a proportion of patients unattended at earlier stage of the disease. For instance, a hospital-based study in Malawi has shown that $10 \%$ of patients with a history of a cough for less than three weeks had smear positive and culture positive TB compared to $22 \%$ who had a cough for more than three weeks (Banda et al, 1998). This suggests that, the current cut off point of a cough for more than three weeks to start screening for TB seem to be unrealistic.

The number of patients being diagnosed as having smear negative pulmonary tuberculosis has been increasing (Parry et al., 1995). The increase is due to several factors including the difficulty of patients to provide adequate early morning sputum specimens, quality of smear, time spent in examining the slide and co-infection with HIV / AIDS (Mendelson, 2007).

Tuberculosis diagnosis based on microscopy of smears made directly from sputum has a low sensitivity especially when performed in overburdened control programmes (Aber et al., 1980). Studies have already shown that liquefaction of sputum with sodium hypochlorite $(\mathrm{NaOCl})$ and concentration of bacilli through

*Correspondence: Dr. Williams Makunde; E-mail: hwmakunde@hotmail.com 
centrifugation increases the sensitivity of direct microscopy (Corper \& Nelson, 1949; Gebre et al., 1995; Miörner et al., 1996). Using this method, the case detection rate may be increased by more than 100\% (Gebre et al., 1995). It has been observed that, the sensitivity of acid-fast smears is directly related to the relative centrifugal force achieved while concentrating the specimen (Rickman et al., 1980).

The use of sodium hypochlorite is ideal since it is cheap and available as household bleach, and also inactivates HIV and M. tuberculosis which could reduce the rate of nosocomial infections in laboratory workers (Rattan et al., 1994). However the bleach method is not widely used because sample preparation is laborious and requires access to centrifuge (Gebre et al., 1995). On the other hand, when sputum induction was carried out, there was an increased smear positive TB rates by $20 \%$ (Parry et al., 1995). In this study, we evaluated an improved method for sputum microscopy, which is based on liquefaction of the sample with household bleach, followed by its concentration by centrifugation prior to staining and microscopy. The bleach microscopy method was compared to standard direct microscopy.

\section{Materials and Methods}

\section{Study population and design}

The study was conducted at Bombo Regional Hospital in Tanga, north-eastern Tanzania between April and May 2004. The outpatient department (OPD) of the hospital serves 40-80 patients a day of which 8-14 are TB suspects. The annual number of TB notification for the entire region is about $3852(\mathrm{MoH}, 2005)$.

Study subjects were patients with cough attending the OPD. Patients were recruited in two groups: Group I consisted of patients referred to the investigator with a cough for less than 3 weeks from the OPD. This group submitted an "on the spot" sputum to be stained using the bleach method. Group II, consisted of screening consecutive TB suspects with a cough for $\geq 3$ weeks using the bleach method and standard Ziel-Neelsen (ZN) method. Patients who qualified for inclusion criteria were examined and had their blood pressures, heart rates, respiratory rates, heart sounds, heights and body weights recorded.

Inclusion criteria were self referred adult patients aged 15 years and above attending
Bombo Regional Hospital from 8:00-13:00hr between Monday and Friday. Patients recruited were those with a history of cough for less than three weeks and more than three weeks. Exclusion was made for patients aged less than 15 years or those who had received antituberculosis chemotherapy within period preceding three months to the commencement of the study.

The sample size was calculated by Epi Info 6 on the assumption that the prevalence of smear positive TB in cough $<3$ weeks is $10 \%$ a sample size of 94 patients to give a $95 \% \mathrm{CI}$ of $\pm 7 \%$ was required. The prevalence of smear-positive TB in cough $\geq 3$ weeks was assumed to be $22 \%$; thus a sample size of 136 patients to give a $95 \%$ $\mathrm{CI}$ of $\pm 5 \%$ was required.

\section{Sputum submission and processing}

Patients were asked to produce an "on spot" sputum specimen in a $20 \mathrm{ml}$ screw plastic container (Cheesbrough, 1985) under the supervision of a trained nurse in the open air. An explanation on how to produce a good quality sputum sample with sufficient volume of sputum was given before collecting the sample. If a sputum sample was not produced within 15 minutes the patient was excluded from the study and was asked to consult the clinical officer for further management. Those produced a sputum sample were asked to return the following day for their results. Those who did not return were recorded as defaulters.

One smear from an "on the spot" specimen was prepared directly from the sputum; air dried and was stained with the routine $\mathrm{ZN}$ method. The rest of the sputum was treated with household bleach (Gebre et al., 1995; Rickman \& Moyer, 1980). $2 \mathrm{ml}$ of sputum was mixed with equal volume of $3-5 \%$ sodium hypochlorite in a plastic falcon tube of $12 \mathrm{ml}$. The sample was left to incubate at room temperature and shaken intermittently for $15 \mathrm{~min}$. before the addition of $6 \mathrm{ml}$ of distilled water to top it up to $10 \mathrm{ml}$. The tube was then covered with parafilm paper before it was centrifuged at 3000 x g for 15 min (Cheesbrough, 1985; Gebre et al., 1995). The supernatant was removed and the pellet re-suspended in a few drops of the remaining fluid. A smear was prepared from the suspension and air-dried. A similar procedure was used to prepare sample from patients with cough for less than three weeks. All ZN stained 
smears were examined semi-quantitatively using bright field microscopy at 1000x magnification.

\section{Data analysis}

Data was entered twice and cleaned using Epi-info version 6 (CDC, Atlanta, GA, USA). Statistical analysis was carried out using Chi-squired test with Yates correction and student $t$-test for normally distributed data and non-parametric tests for data not normally distributed.

\section{Ethical consideration}

This study was approved by the Ethical Committee of the National Institute for Medical Research, Tanzania. Patients were asked whether they wish to participate in this study before enrolment and an oral informed consent was obtained from each participant. Patients who had cough for $>3$ weeks with no acid fast bacilli (AFB) in their smear were treated with Cotrimoxazole for ten days and asked to return back. If after ten days they were still coughing, they were sent to the clinical officer for routine screening procedures. Those patients identified to have positive smear were directly sent to the TB coordinator for appropriate anti-TB chemotherapy. However, those patients with cough for three or more weeks found to have no AFB in their smear were sent to a clinic for further investigations according to the national TB control programme policy. in Group II did not complete the study and therefore were not included in the analysis. The mean age of the patients was 34.9 years and this included 34.0 years in Group I and 35.3 years in Group II. Sixty-seven (59.3\%) of the patients were illiterate. Males and females accounted for 55\% and $45 \%$ of the patients, respectively. Only $49.5 \%$ of the patients had formal occupation.

Using ZT technique, tuberculosis was identified in $13.8 \%$ and $23.8 \%$ of the patients with history of cough for $<3$ and $\geq 3$, respectively (Table 1). When using a bleach technique, tuberculosis was diagnosed in $28.3 \%$ of the patients with history of $\geq 3$ week cough.

In Group II, $110(97.3 \%)$ patients submitted the "on the spot" sputum specimens, $3(2.6 \%)$ patients were "unable". A total of 109 (93.2\%) patients had submitted the early morning sputum specimen while 106 (90.6) produced the "on the spot" sputum specimen. Twenty-seven (23.8\%) patients were smear-positive by the three smear case-finding strategy, while $32(28.3 \%)$ were smear-positive by the bleach method in Group II patients. Those with negative smear in Group II were $81(71.7 \%)$. In Group I, $8(13.8 \%)$ patients were considered to be smear positive after been independently assessed by the technician using a traditional Ziel-Neelsen staining technique. On the other hand $50(86.2 \%)$ were negative.

The significant risk factors associated

Table 1: Tuberculosis cases detected in patients with cough for $<3$ and $\geq 3$ weeks

\begin{tabular}{lcccc}
\hline Duration of cough & Positive & Negative & Total & 95\% CI \\
\hline Cough $<3$ weeks & $8(13.8 \%)$ & $50(86.2 \%)$ & 58 & $5-23$ \\
Cough $\geq 3$ weeks & $27(23.9 \%)$ & $86(76.1 \%)$ & 113 & $13-28$ \\
Cough $\geq 3$ weeks by bleach single smear & $32(28.3 \%)$ & $81(71.7 \%)$ & 113 & $20-36$ \\
\hline
\end{tabular}

\section{Results}

Smear positive was defined as more than 10 acidfast bacilli (AFB) in 100 fields in more than two "on spot" smears. Smear negative was defined as those with no AFB in 100 fields in all three smears. On the other hand, indeterminate was defined as 1-9 AFBs in 100 fields in 1 smear.

A total 175 patients were recruited, however, only $171(97.7 \%)$ were covered in this analysis. Of these, 58 (33.1\%) were included in Group I and 117 in Group II. Four patients with smear-positive identified in this study were weight loss, haemoptysis, body mass index (BMI) and increased respiratory rate (Table 2). However, low BMI, gender, age and having received treatment for cough previously, and increased pulse rate were not statistically associated with smear-positivity. Although cough was the leading symptom in both groups, increased respiratory rate was almost similar in the two groups. However, majority of patients in Group II had more days (46.02 days) of cough than those in Group I (12.34 days). 
Table 2: Risk factors associated with smear positive when considering bleach method alone

\begin{tabular}{|c|c|c|c|c|c|c|c|}
\hline \multirow[t]{2}{*}{ Variable } & & \multicolumn{2}{|c|}{ Smear positive } & \multicolumn{2}{|c|}{ Smear negative } & \multirow[t]{2}{*}{$P$-value } & \multirow{2}{*}{$\begin{array}{l}\text { Odds ratio (level of } \\
\text { significance) }\end{array}$} \\
\hline & & $\mathrm{N}$ & $\%$ & & $\%$ & & \\
\hline \multirow[t]{2}{*}{ Sex } & Female & 14 & 40.0 & 63 & 49.6 & & \\
\hline & Male & 24 & 60.0 & 64 & 50.4 & & \\
\hline Mean age & & 31 & 12.04 & 35 & 13.2 & 0.7 & \\
\hline Body mass index & & 17.12 & 2.4 & 19.19 & 2.7 & 0.0003 & \\
\hline \multirow[t]{2}{*}{ Weight loss } & Yes & 35 & 87.5 & 67 & 53.0 & 0.0004 & \\
\hline & No & 5 & 12.5 & 60 & 47.2 & & \\
\hline \multirow[t]{2}{*}{ Haemoptysis } & Yes & 15 & 37.5 & 21 & 16.5 & 0.003 & \\
\hline & No & 25 & 62.5 & 106 & 83.5 & & \\
\hline \multirow[t]{2}{*}{ Cough } & Yes & 16 & 40.0 & 43 & 33.9 & & $1.3(0.58-2.9)$ \\
\hline & No & 24 & 60.0 & 84 & 66.1 & & \\
\hline \multirow[t]{2}{*}{ Chest pain } & Yes & 14 & 35.0 & 58 & 45.7 & & $1.04(0.28-1.43)$ \\
\hline & No & 26 & 65.0 & 69 & 54.3 & & \\
\hline \multirow[t]{2}{*}{ Cough treatment } & Yes & 29 & 72.5 & 98 & 77.2 & & $1.47(0.67-3.26)$ \\
\hline & No & 11 & 27.5 & 29 & 22.8 & & \\
\hline \multicolumn{2}{|c|}{ Mean respiratory rate } & 24 & & 21 & & 0.0001 & \\
\hline
\end{tabular}

The characteristics of the patients are summarised in Table 3. The number of patients who had attended elsewhere was 78 of which 3 were in Group I and 75 were in Group II. The TB smear
The mean body weight and height of the patients was $47.6 \mathrm{~kg}(\mathrm{SD}=9.2)$ and $1.595 \mathrm{~m}$, respectively and was similar in both groups. Mean respiratory rate and heart rate were 22.129 and 79.848 per

Table 3: Subjective characteristics of the study population shown as number (\%) of patients

\begin{tabular}{llll}
\hline Symptoms & Cough $<3$ weeks & Cough $>3$ weeks & Total \\
\hline Cough & $21(36.2 \%)$ & $39(33.6 \%)$ & $60(34.5 \%)$ \\
Attend elsewhere & $3(5.2 \%)$ & $75(66.4 \%)$ & $78(69 \%)$ \\
Weight loss & $22(38 \%)$ & $84(74.3 \%)$ & $106(62 \%)$ \\
Haemoptysis & $12(20.7 \%)$ & $24(41.4 \%)$ & $36(21.1 \%)$ \\
Fever & $49(84.5 \%)$ & $80(71.8 \%)$ & $129(75.4 \%)$ \\
Chest pain & $17(29.3 \%)$ & $57(50.4 \%)$ & $74(43.3 \%)$ \\
Night sweats & $16(27.6 \%)$ & $56(49.6 \%)$ & $72(42.1 \%)$ \\
Short of breath & $28(48.3 \%)$ & $70(62 \%)$ & $98(57.3 \%)$ \\
TB contact & $10(17.2 \%)$ & $18(16 \%)$ & $28(16.4 \%)$ \\
\hline
\end{tabular}

positive cases detected in group II patient by a single bleach smear was $32(28.3 \%)$ with $(95 \%$ $\mathrm{CI}=20-36)$ as compared to those in Group II 27 $(23.8 \%)$ with $(95 \% \mathrm{CI}=13-28)$. minute, respectively. More patients in Group II were found with enlargement of lymph node, skin lesions and oral thrush (Table 4). 
Table 4: Objective characteristics of the study population as shown by number of patients (\%)

\begin{tabular}{llll}
\hline Character & $\begin{array}{l}\mathbf{n = 5 8} \\
\text { Cough }<3 \text { weeks }\end{array}$ & $\begin{array}{l}\mathbf{N = 1 1 3} \\
\text { Cough }>\text { 3 weeks }\end{array}$ & $\begin{array}{l}\mathbf{N = 1 7 1} \\
\text { Total }\end{array}$ \\
\hline Oral thrush & $4(6.9 \%)$ & $22(19.5 \%)$ & $26(15.2 \%)$ \\
Skin lesions & $5(8.6 \%)$ & $19(16.8 \%)$ & $24(14.0 \%)$ \\
Lymph node enlargement & $9(15.5 \%)$ & $48(42.5 \%)$ & $57(50.4 \%)$ \\
X-ray done & $7(12.1 \%)$ & $26(23.0 \%)$ & $33(19.3 \%)$ \\
\hline
\end{tabular}

When comparing a single bleach smear microscopy with three standards ZN smear in Group II patients, bleach smear positive were 26 $(81.3 \%)$ and indeterminate $6(18.8 \%)$. The bleach smear negative was $100 \%$ (77), indeterminate smear positive $1(25.0 \%)$ and smear negative $2(50.0 \%)$. The sensitivity and specificity were $100 \%$ [95\% CI=100\%], Positive Predictive Value (PPV) was $88.9 \%$ (95\% CI 79\%-99\%) and Negative Predictive Value (NPV) was 95.1\% (95\% CI 91$100 \%$ ). The bleach result positive smears were 29 $(100.0 \%)$, while negative smears were $3(3.6 \%)$. Both the sensitivity and specificity were $100 \%$. PPV was $90.6 \%$ (95\% CI 81\%-100\%) while NPV was $100 \%$ (95\% CI 94\%-98\%).

\section{Discussion}

The prevalence of smear-positive TB in patients with a cough of less than three weeks was similar to that reported in Malawi (Harries et al., 1997). The proportion of smear-positive disease detected in this study was found in the cough of less than three weeks that is not normally considered as a TB suspect group. These patients are infectious TB patients who would not have routinely been detected. It is not known whether these smearpositive cases would have returned to health care facilities later on. All the smear-positive TB patients detected in the short duration group by a single bleached smear were also found to be independently positive by a single $\mathrm{ZN}$ smear. Facilities for Mycobacterium culture as in many developing world are not available, and therefore cultures are not routinely performed. However, a previous study (Banda et al, 1998) has shown that, 35\% smear-positive patients with a cough for less than three weeks had pulmonary tuberculosis. Although cultures are not routinely carried out in majority of the developing countries, when performed over $99 \%$ of the smear positive cases turn to be culture positive.
Direct smear microscopy does not discriminate between tubercle bacilli and other mycobacteria, however this is not a major problem in developing world because majority of patients with AFB in sputum smears have TB as atypical mycobacteria does not present in sufficient concentration in sputum to be detected by direct microscopy (Lipsky et al., 1984). It is also possible that the direct smear microscopy may be a false positive result because of contamination by environmental bacteria or cross-contamination.

In this study, the short duration group did not statistically differ from the long duration group with respect to age, sex and previous TB contact. On the other hand having less period of illness, less visits elsewhere; less being treated for cough previously correlated with cough for $<3$ weeks than the long duration cough. This would appear to support a shorter illness period and subjectively verify the history of a cough for less than three weeks.

Many patients with long duration cough failed to submit all three sputum samples. This led to $12.4 \%$ patients being classified as indeterminate. However with the bleach method only a few $(3.5 \%)$ were classified as indeterminate using the International Union Against Tuberculosis and Lung Diseases definition for smear-positivity and negativity. The bleach method increased smear-positivity by $13 \%$ with $>100 \%$ sensitivity and specificity. Previous studies found increased smear-positivity by $14 \%$ with $100 \%$ sensitivity and specificity (Allwood et al., 1997). Possibly it is in this group where a high number of patients presented with oral thrush were found. In most cases oral thrush is associated with immune suppression demonstrating some of these patients could have contacted HIV infection.

The traditional approach of waiting for three sputum smear samples before any of them is examined leads to delay in diagnosis and treatment. Previous studies have shown 
an increased risk of nosocomial transmission of tuberculosis in hospital workers (Harries et al, 1997, 1998) due to the current procedure of waiting for three sputum smears. The findings of this study suggest that, bleach method could widely be applied as routine procedure in all laboratories that perform direct microscopy only and its use would definitely lower the risk of laboratory infection since sodium hypochlorite is a potent disinfectant and accelerate patient prompt treatment. Moreover the rapid identification of smear-positive TB has the potential to increase patient access to diagnosis and treatment and significantly improve the efficiency of passive case finding both in economic and human terms. It is obvious that patients with a short history of cough would benefit from pulmonary tuberculosis screening strategies with sputum examination at an early stage of less than the common three week period. Moreover, with the high sensitivity and specificity of the bleached sputum microscopy method, it is time to revisit the policy regarding tuberculosis screening in Tanzania.

\section{Acknowledgements}

We are grateful to the study participants for their willingness and cooperation during the study period. Messrs E. Anga, A. Mushi and the late J. Tao of the Bombo Hospital, Tanga are thanked for their excellent technical assistance. Dr. Andrew Y. Kitua, Director General, National Institute for Medical Research, Tanzania is thanked for the permission to publish.

\section{References}

Aber, V.R., Allen, R.B.W., Mitchison, D.A., Ayuma, P., Edwards, E.A. \& Keyes, A.B. (1980) Quality control in tuberculosis bacteriology. 1. Laboratory studies on isolated positive cultures and the efficiency of direct smear examination. Tubercle 61, 123-133.

Allwood, M.D, Lee, Y.C., Salaniponi, F.M., Kuppusamy, I. \& Squire, S.B. (1997) Bleach treatment of a single sputum sample could improve tuberculosis case finding. FIS Abstracts. Journal of Infections 34, 163.

Banda, H.T., Harries, A.D., Welby, S., Boeree, M.J., Wirima, J.J., Subramanyam, V.R.,
Maher, D. \& Nunn, P.A. (1998) Prevalence of tuberculosis in TB suspects with short duration of cough. Transactions of the Royal Society of Tropical Medicine and Hygiene 92, 161-163.

Cheesbrough, M. (1985) Medical Laboratory Manual for Tropical Countries. Vol 11, Microbiology 105, 294-297. Uni Press, Cambridge.

Corper, H.J. \& Nelson, C.R. (1949) Methods for concentrating acid-fast bacilli. American Journal of Clinical Pathology 19, 269-273.

Gebre, N., Karlsson, U. Johnson, G., Makaden, R., Wolde, A., Assefa, A. \& Miorner, H. (1995) Improved microscopical diagnosis of PTB in developing countries. Transaction of the Royal Society of Tropical Medicine and Hygiene 89, 191-193.

Harries, A.D., Kamenya, A., Namarika, D., Msolomba, I.W., Salaniponi, F.M., Nyangulu, D.S. \& Nunn, P. (1997) Delays in diagnosis and treatment of smearpositive tuberculosis and the incidence of tuberculosis in hospital nurses in Blantyre Malawi. Transaction of the Royal Society of Tropical Medicine and Hygiene 91, 15-17.

Harries, A.D., Kamenya, A., Subramanyam, V.R., Maher, D., Squire, S.B., Wirima, J.J., Nyangulu, D.S. \& Nunn, P. (1997) Screening pulmonary tuberculosis suspects in Malawi: testing different strategies. Transaction of the Royal Society of Tropical Medicine and Hygiene 91, 416419.

Harries, A.D., Nyirenda, J.E., Benerjee, A., Mundy, C. \& Salaniponi, F.M. (1998) District sputum smear microscopy services in Malawi. International Union Against Tuberculosis and Lung Disease 2, 914-918.

L'Herminez, R.H. (1993) Urgent need for a new approach to the diagnosis of tuberculosis in developing countries in the decade of AIDS. Tropical and Geographical Medicine 45, 145-149.

Lipsky, B.A., Gates, J.A., Tenover, F.C. \& Plorde, J.J. (1984) Factors affecting the clinical value of microscopy for acid-fast bacilli. Revision of Infectious Diseases 4, 214-222.

Mendelson, M. (2007) Diagnosis of tuberculosis in HIV-infected patients: challenges and future prospects. British Medical Bulletin 0, 1dm009V1-17. 
Mirner, H., Ganlov, G., Yahannes, Z. \& Adane, Y. (1996) Improved sensitivity of direct microscopy for acid-fast bacilli: sedimentation as an alternative to centrifugation for concentration of tubercle bacilli. Journal of Clinical Microbiology 34, 3206-3207.

Parry, C.M., Kazimoto, O., Harries, A., Wirima, J., Nyirinda, C., Nyangulu, D. \& Hart, C.A. (1995) The use of sputum induction for establishing a diagnosis in patients with suspected pulmonary tuberculosis in Malawi. Tubercle Journal 76, 72-76.
Rattan, A., Kishore, K., Singh, S., Jaber, M., Xess, I. \& Kumar, R. (1994) A safe sputum processing method for detection of tuberculosis in developing countries. Journal of Clinical Microbiology 47, 411413.

Rickman, T. \& Moyer, N. (1980) Increased sensitivity of acid fast smears. Journal of Clinical Microbiology 11, 618-620.

WHO (2006) The Global Plan to Stop TB 2006-2015. The World Health Organization, Geneva. http: / / www.stoptb.org / globalplan. (Accessed on 27th July 2006). 